\title{
Editorial: Advancements in Technology-Based Assessment: Emerging Item Formats, Test Designs, and Data Sources
}

\author{
Frank Goldhammer $^{1,2}$, Ronny Scherer ${ }^{3}$, Samuel Greiff ${ }^{4}$ \\ ${ }^{1}$ DIPF | Leibniz Institute for Research and Information in Education \\ ${ }^{2}$ Centre for International Student Assessment (ZIB) \\ ${ }^{3}$ University of Oslo, Centre for Educational Measurement (CEMO) \\ ${ }^{4}$ University of Luxembourg
}

Technology has become an indispensable tool for educational and psychological assessment in today’s world. Individual researchers and large-scale assessment programs alike are increasingly using digital technology (e.g., laptops, tablets, and smartphones) to collect behavioral data beyond the mere correctness of item responses. Along these lines, technology innovates and enhances assessments in terms of item and test design, methods of test delivery, data collection and analysis, and the reporting of test results.

The aim of this research topic is to present recent developments in technology-based assessment and in the advancements of knowledge associated with it. Our focus is on cognitive assessments, including the measurement of abilities, competences, knowledge, and skills, but also includes non-cognitive aspects of assessment (Rausch, Kögler, \& Seifried, 2019; Simmering, Ou, \& Bolsinova, 2019). In the area of (cognitive) assessments, the innovations driven by technology are manifold, and the topics covered in this collection are, accordingly, wide and comprehensive: Digital assessments facilitate the creation of new types of stimuli and response formats that were out of reach for assessments using paper; for 
instance, interactive simulations may include multimedia elements, as well as virtual or augmented realities (Cipresso et al., 2019; De-Juan-Ripoli et al., 2019). These types of assessments also allow for the widening of the construct coverage in an assessment; for instance, through stimulating and making visible certain problem-solving strategies that represent new forms of problem solving (Han, He, \& von Davier, 2019; Kroeze, van den Berg, Lazonder, Veldkamp, \& de Jong, 2019). Moreover, technology allows for the automated generation of items based on specific item models (Shin, Guo, \& Gierl, 2019). Such items can be assembled into tests in a more flexible way than what is possible in paperand-pencil tests and can even be created on the fly; for instance, tailoring item difficulty to individual ability (adaptive testing) while assuring that multiple content constraints are met (Born, Fink, Spoden, \& Frey, 2019; Zhang, Wang, Gao, Cai, \& Tu, 2019). As a requirement for adaptive testing, or to lower the burden of raters who code item responses manually, computers enable the automatic scoring of constructed responses; for instance, text responses can be coded automatically by using natural language processing and text mining (He, Veldkamp, Glas, \& van den Berg, 2019; Horbach \& Zesch, 2019).

Technology-based assessments provide not only response data (e.g., correct vs. incorrect responses) but also process data (e.g., frequencies and sequences of test-taking strategies, including navigation behavior) that reflect the course of solving a test item and gives information on the path towards the solution (Han et al., 2019). Process data, among others, have been used successfully to evaluate and explain data quality (Lindner, Lüdtke, \& Nagy, 2019), to define process-oriented latent variables (De Boeck \& Scalise, 2019), to improve measurement precision, and to address substantial research questions (Naumann, 2019). Large-scale result and process data also call for data-driven computational approaches in addition to traditional psychometrics and new concepts for storing and managing data (Von Davier, Wong, Polyak, \& Yudelson, 2019). 
The contributions of this research topic address how technology can further improve and enhance educational and psychological assessment from various perspectives. Regarding educational testing, not only is research presented on the assessment of learning, that is, the summative assessment of learning outcomes (Molnár \& Csapó, 2019), but a number of studies on this topic also focus conceptually and empirically on the assessment for learning, that is, the formative assessment providing feedback to support the learning process (ArieliAttali, Ward, Thomas, Deonovic, \& von Davier, 2019; Blaauw, van der Gaag, Snell, Emerencia, Kunnen, \& Jonge, 2019; Csapó \& Molnár, 2019; Den Ouden, Keuning, \& Eggen, 2019; Kroeze et al., 2019).

Table 1 gives an overview of all the papers included in this research topic and summarizes them with respect to their key features. Reflecting the scope of the research topic, we used four major categories to classify the papers: (1) papers focusing on the use of new data types and sources, (2) innovative item designs, (3) innovative test designs, and (4) statistical approaches. We refrained from multiple category assignments of papers, which was possible, and focused on their core contribution. The papers' key findings and advancements impressively represent the current state-of-the-art in the field of technology-based assessment in (standardized) educational testing, and, as topic editors, we were happy to receive such a great collection of papers with various foci.

Regarding the future of technology-based assessment, we assume that inferences about the individual's or learner's knowledge, skills, or other attributes will increasingly be based on empirical (multimodal) data from less- or non-standardized testing situations. Typical examples are stealth assessments in digital games (Shute, 2015; Shute \& Ventura, 2013), digital learning environments (Nguyen, Huptych, \& Rienties, 2018), or online activities (Kosinski, Stillwell, \& Graepel, 2013). Such new kinds of unobtrusive, continuous assessments will further extend the traditional assessment paradigm and enhance our 
understanding of what an item, a test, and the empirical evidence for inferring attributes can be (Mislevy, 2019). Major challenges lie in the identification and synthesis of evidence from the situations the individual encounters in these non-standardized settings, as well as in validating the interpretation of derived measures. This research topic provides much input for these questions. We hope that you will enjoy reading the contributions as much as we did. 


\section{References}

Kosinski, M., Stillwell, D., \& Graepel, T. (2013). Private traits and attributes are predictable from digital records of human behavior. Proceedings of the National Academy of Sciences, 110(15), 5802. doi:10.1073/pnas.1218772110

Mislevy, R. (2019). On integrating psychometrics and learning analytics in complex assessments. In H. Jiao, R. W. Lissitz, \& A. van Wie (Eds.), Data analytics and psychometrics (pp. 1-52). Charlotte, NC, USA Information Age Publishing.

Nguyen, Q., Huptych, M., \& Rienties, B. (2018). Linking students' timing of engagement to learning design and academic performance. Paper presented at the Proceedings of the 8th International Conference on Learning Analytics and Knowledge, Sydney, New South Wales, Australia.

Shute, V. (2015). Stealth assessment. In J. Spector (Ed.), The SAGE Encyclopedia of Educational Technology (pp. 675-676). Thousand Oaks, California: SAGE Publications, Inc.

Shute, V., \& Ventura, M. (2013). Stealth Assessment: Measuring and Supporting Learning in Video Games: The MIT Press. 
Table 1

Overview of the papers.

\begin{tabular}{|c|c|c|c|c|c|c|}
\hline Article & $\begin{array}{l}\text { Area(s) of } \\
\text { advancement }\end{array}$ & Data types & $\begin{array}{l}\text { Statistical } \\
\text { approach }\end{array}$ & $\begin{array}{l}\text { Assessment } \\
\text { purpose } \\
\text { (of/for } \\
\text { learning) }\end{array}$ & $\begin{array}{l}\text { Assessment } \\
\text { domains }\end{array}$ & Key finding and advancement \\
\hline \multicolumn{7}{|c|}{ Focus on new data types and sources } \\
\hline $\begin{array}{l}\text { Blaauw et } \\
\text { al. (2019) }\end{array}$ & $\begin{array}{l}\text { Computerized } \\
\text { assessment of } \\
\text { learning with } \\
\text { multiple } \\
\text { informants }\end{array}$ & $\begin{array}{l}\text { Survey } \\
\text { responses, } \\
\text { platform } \\
\text { user data }\end{array}$ & $\begin{array}{l}\text { Descriptive } \\
\text { approach }\end{array}$ & FOR & $\begin{array}{l}\text { Vocational } \\
\text { education }\end{array}$ & $\begin{array}{l}\text { Multi-informant time-series data } \\
\text { can inform the success of } \\
\text { educational interventions to } \\
\text { support students at risk. }\end{array}$ \\
\hline $\begin{array}{l}\text { De Boeck } \\
\text { \& Scalise } \\
(2019)\end{array}$ & $\begin{array}{l}\text { Log-file and } \\
\text { performance } \\
\text { data to assess } \\
\text { ColPS }\end{array}$ & $\begin{array}{l}\text { Actions, } \\
\text { response } \\
\text { times, } \\
\text { correctness } \\
\text { of item } \\
\text { responses }\end{array}$ & $\begin{array}{l}\text { Confirmatory } \\
\text { factor } \\
\text { analysis }\end{array}$ & OF & $\begin{array}{l}\text { Collaborativ } \\
\text { e problem } \\
\text { solving } \\
\text { (PISA } \\
\text { 2015) }\end{array}$ & $\begin{array}{l}\text { Dependencies among action, } \\
\text { time-on task, and performance } \\
\text { indicators do not only exist at the } \\
\text { construct but also the item } \\
\text { (residual) level. }\end{array}$ \\
\hline $\begin{array}{l}\text { Lindner et } \\
\text { al. (2019) }\end{array}$ & $\begin{array}{l}\text { Time-on task } \\
\text { to identify } \\
\text { rapid guessing }\end{array}$ & $\begin{array}{l}\text { Correctness } \\
\text { of item } \\
\text { responses, } \\
\text { response } \\
\text { times }\end{array}$ & $\begin{array}{l}\text { Latent class } \\
\text { analysis }\end{array}$ & OF & $\begin{array}{l}\text { Science } \\
\text { achievemen } \\
\mathrm{t}\end{array}$ & $\begin{array}{l}\text { Response times can provide } \\
\text { information about rapid-guessing } \\
\text { behavior and its relations to } \\
\text { cognitive resources and test- } \\
\text { taking effort. }\end{array}$ \\
\hline $\begin{array}{l}\text { Naumann } \\
\text { (2019) }\end{array}$ & $\begin{array}{l}\text { Time-on task } \\
\text { data of reading }\end{array}$ & $\begin{array}{l}\text { Correctness } \\
\text { of item } \\
\text { responses, } \\
\text { response }\end{array}$ & $\begin{array}{l}\text { Linear mixed } \\
\text { modeling }\end{array}$ & OF & $\begin{array}{l}\text { Reading } \\
\text { literacy } \\
\text { (PISA } \\
\text { 2009) }\end{array}$ & $\begin{array}{l}\text { Response times can help identify } \\
\text { relations between item } \\
\text { difficulties, strategic knowledge, } \\
\text { skills, and motivation to } \\
\text { ultimately craft a validity }\end{array}$ \\
\hline
\end{tabular}




\begin{tabular}{|c|c|c|c|c|c|c|}
\hline \multicolumn{6}{|c|}{ times } & \multirow{2}{*}{$\begin{array}{l}\text { argument. } \\
\text { Challenges and limitations in } \\
\text { using technology-enhanced } \\
\text { assessments require } \\
\text { consideration. }\end{array}$} \\
\hline $\begin{array}{l}\text { Simmerin } \\
\text { g et al. } \\
(2019)\end{array}$ & $\begin{array}{l}\text { Assessment of } \\
\text { non-cognitive } \\
\text { skills }\end{array}$ & $\begin{array}{l}\text { Continuous } \\
\text { process data } \\
\text { (e.g., } \\
\text { behavioral, } \\
\text { physiological) }\end{array}$ & - & - & $\begin{array}{l}\text { Non- } \\
\text { cognitive } \\
\text { skills }\end{array}$ & \\
\hline $\begin{array}{l}\text { Von } \\
\text { Davier et } \\
\text { al. (2019) }\end{array}$ & $\begin{array}{l}\text { Data } \\
\text { paradigms for } \\
\text { educational } \\
\text { learning and } \\
\text { assessment } \\
\text { systems }\end{array}$ & $\begin{array}{ll}\text { Response } & \text { e } \\
\text { behavior, } & \text { le } \\
\text { test content, } & \\
\text { instructional } \\
\text { content }\end{array}$ & $\begin{array}{l}\text { e.g., machine } \\
\text { learning }\end{array}$ & OF/FOR & Divers & $\begin{array}{l}\text { The concept of the "data cube" } \\
\text { can be used to label, collect and } \\
\text { store data. }\end{array}$ \\
\hline \multicolumn{7}{|c|}{ Focus on innovative item designs } \\
\hline $\begin{array}{l}\text { Arieli- } \\
\text { Attali et } \\
\text { al. (2019) }\end{array}$ & $\begin{array}{l}\text { Learning } \\
\text { design }\end{array}$ & $\begin{array}{l}\text { Learners' } \\
\text { responses and } \\
\text { use of learning } \\
\text { support }\end{array}$ & $\begin{array}{l}\text { e.g., } \\
\text { hidden } \\
\text { Markov } \\
\text { modelling }\end{array}$ & FOR & Divers & $\begin{array}{l}\text { The traditional evidence centered } \\
\text { design models can be expanded } \\
\text { to assess learning. }\end{array}$ \\
\hline $\begin{array}{l}\text { Cipresso } \\
\text { et al. } \\
(2019)\end{array}$ & $\begin{array}{l}\text { Assessment of } \\
\text { unilateral } \\
\text { spatial neglect }\end{array}$ & $\begin{array}{l}\text { Correctness of } \\
\text { item responses }\end{array}$ & - & - & $\begin{array}{l}\text { Unilateral } \\
\text { spatial } \\
\text { neglect }\end{array}$ & $\begin{array}{l}\text { Complex 3D environments on } \\
\text { mobile devices are promising for } \\
\text { the ecological assessment of } \\
\text { unilateral spatial neglect. }\end{array}$ \\
\hline $\begin{array}{l}\text { De-Juan- } \\
\text { Ripoli et } \\
\text { al. (2019) }\end{array}$ & $\begin{array}{l}\text { Assessment of } \\
\text { risk taking }\end{array}$ & $\begin{array}{l}\text { Behavioral and } \\
\text { physiological } \\
\text { responses }\end{array}$ & - & - & Risk taking & $\begin{array}{l}\text { Virtual realities (VR) can be } \\
\text { employed to simulate hazardous } \\
\text { situations realistically. }\end{array}$ \\
\hline $\begin{array}{l}\text { Den } \\
\text { Ouden et } \\
\text { al. (2019) }\end{array}$ & $\begin{array}{l}\text { Computerized } \\
\text { dynamic } \\
\text { assessment of } \\
\text { text }\end{array}$ & $\begin{array}{l}\text { Correctness of } \\
\text { item responses }\end{array}$ & $\begin{array}{l}\text { Linear } \\
\text { modeling } \\
\text { and }\end{array}$ & FOR & $\begin{array}{l}\text { Text } \\
\text { comprehens } \\
\text { ion }\end{array}$ & $\begin{array}{l}\text { Computer-based dynamic } \\
\text { assessments bear the potential to } \\
\text { support students in acquiring }\end{array}$ \\
\hline
\end{tabular}




\begin{tabular}{|c|c|c|c|c|c|c|}
\hline & $\begin{array}{l}\text { comprehension } \\
\text { skills }\end{array}$ & & MTMM & & & reading skills. \\
\hline $\begin{array}{l}\text { Horbach } \\
\text { \& Zesch } \\
(2019)\end{array}$ & $\begin{array}{l}\text { Automated } \\
\text { content scoring }\end{array}$ & Written text & $\begin{array}{l}\text { Machine } \\
\text { learning }\end{array}$ & $\mathrm{OF}$ & Diverse & $\begin{array}{l}\text { Automated content scoring } \\
\text { approaches can take into account } \\
\text { the variance in learner answers. }\end{array}$ \\
\hline $\begin{array}{l}\text { Kroeze et } \\
\text { al. (2019) }\end{array}$ & $\begin{array}{l}\text { Automated } \\
\text { feedback } \\
\text { generation }\end{array}$ & $\begin{array}{l}\text { Written text, } \\
\text { actions, } \\
\text { correctness of } \\
\text { item responses }\end{array}$ & $\begin{array}{l}\text { Descriptiv } \\
\text { e } \\
\text { approach, } \\
\text { linear } \\
\text { model }\end{array}$ & OF/FOR & $\begin{array}{l}\text { Scientific } \\
\text { inquiry in } \\
\text { economics } \\
\text { and physics }\end{array}$ & $\begin{array}{l}\text { Automated feedback on scientific } \\
\text { hypotheses can agree with human } \\
\text { ratings to a great extent, and } \\
\text { students who receive it are likely } \\
\text { to develop better hypotheses than } \\
\text { those who don't. }\end{array}$ \\
\hline \multicolumn{7}{|c|}{ Focus on innovative test designs } \\
\hline $\begin{array}{l}\text { Born et al. } \\
\text { (2019) }\end{array}$ & $\begin{array}{l}\text { Computerized } \\
\text { adaptive } \\
\text { testing and test } \\
\text { equating }\end{array}$ & $\begin{array}{l}\text { Correctness } \\
\text { of item } \\
\text { responses }\end{array}$ & $\begin{array}{l}\text { Item response } \\
\text { theory }\end{array}$ & OF & - & $\begin{array}{l}\text { Equating designs and CAT can } \\
\text { be combined through a } \\
\text { continuous calibration strategy. }\end{array}$ \\
\hline $\begin{array}{l}\text { Csapó \& } \\
\text { Molnár } \\
\text { (2019) }\end{array}$ & $\begin{array}{l}\text { Assessment for } \\
\text { teaching and } \\
\text { learning }\end{array}$ & $\begin{array}{l}\text { Correctness } \\
\text { of item } \\
\text { responses }\end{array}$ & $\begin{array}{l}\text { Item response } \\
\text { theory }\end{array}$ & FOR & $\begin{array}{l}\text { Mathematic } \\
\text { s, science, } \\
\text { and reading }\end{array}$ & $\begin{array}{l}\text { Teaching and learning can be } \\
\text { supported on a large scale by } \\
\text { online assessment solutions } \\
\text { (authoring, assembly, scoring, } \\
\text { delivery, feedback). }\end{array}$ \\
\hline $\begin{array}{l}\text { Molnár \& } \\
\text { Csapó } \\
(2019)\end{array}$ & $\begin{array}{l}\text { Computerized } \\
\text { assessment of } \\
\text { cognitive } \\
\text { development }\end{array}$ & $\begin{array}{l}\text { Correctness } \\
\text { of item } \\
\text { responses }\end{array}$ & $\begin{array}{l}\text { Confirmatory } \\
\text { factor } \\
\text { analysis and } \\
\text { structural } \\
\text { equation } \\
\text { models }\end{array}$ & OF/FOR & $\begin{array}{l}\text { Mathematic } \\
\text { s, science, } \\
\text { and reading } \\
\text { competence } \\
\text { s }\end{array}$ & $\begin{array}{l}\text { Computerized assessments can } \\
\text { capture differences in the } \\
\text { academic performance on tests in } \\
\text { mathematics, science, and } \\
\text { reading across grade levels and } \\
\text { make visible the psychological } \\
\text { dimension of learning. }\end{array}$ \\
\hline
\end{tabular}




\begin{tabular}{|c|c|c|c|c|c|c|}
\hline $\begin{array}{l}\text { Rausch et } \\
\text { al. (2019) }\end{array}$ & $\begin{array}{l}\text { Embedded } \\
\text { experience } \\
\text { sampling for } \\
\text { assessing non- } \\
\text { cognitive skills }\end{array}$ & $\begin{array}{l}\text { Survey } \\
\text { responses, } \\
\text { correctness } \\
\text { of item } \\
\text { responses }\end{array}$ & $\begin{array}{l}\text { MTMM, item } \\
\text { response } \\
\text { theory }\end{array}$ & $\mathrm{OF}$ & $\begin{array}{l}\text { Non- } \\
\text { cognitive } \\
\text { facets of } \\
\text { problem } \\
\text { solving }\end{array}$ & $\begin{array}{l}\text { Embedded experience sampling } \\
\text { provides an approach to assess } \\
\text { non-cognitive facets of } \\
\text { competences through multiple } \\
\text { self-reports. }\end{array}$ \\
\hline $\begin{array}{l}\text { Zhang et } \\
\text { al. (2019) }\end{array}$ & $\begin{array}{l}\text { Computerized } \\
\text { adaptive } \\
\text { testing of } \\
\text { Internet } \\
\text { addiction }\end{array}$ & $\begin{array}{l}\text { Survey } \\
\text { responses }\end{array}$ & $\begin{array}{l}\text { Item response } \\
\text { theory }\end{array}$ & OF & $\begin{array}{l}\text { Internet } \\
\text { addiction }\end{array}$ & $\begin{array}{l}\text { A computerized adaptive test of } \\
\text { Internet addiction assessed the } \\
\text { construct accurately and } \\
\text { efficiently, and provided } \\
\text { evidence for both the reliability } \\
\text { and validity of the resultant test } \\
\text { scores. }\end{array}$ \\
\hline \multicolumn{7}{|c|}{ Focus on statistical approaches } \\
\hline $\begin{array}{l}\text { Han et al. } \\
\text { (2019) }\end{array}$ & $\begin{array}{l}\text { Data mining } \\
\text { using random } \\
\text { forests to } \\
\text { predict item } \\
\text { performance }\end{array}$ & $\begin{array}{l}\text { Actions, } \\
\text { response } \\
\text { times, } \\
\text { correctness } \\
\text { of item } \\
\text { responses }\end{array}$ & $\begin{array}{l}\text { Tree-based } \\
\text { model }\end{array}$ & OF & $\begin{array}{l}\text { Problem } \\
\text { solving } \\
\text { (PISA } \\
\text { 2012) }\end{array}$ & $\begin{array}{l}\text { A random forest algorithm can } \\
\text { generate and select features from } \\
\text { the process data that predict } \\
\text { students' item responses. }\end{array}$ \\
\hline $\begin{array}{l}\text { He et al. } \\
\text { (2019) }\end{array}$ & $\begin{array}{l}\text { Text mining } \\
\text { and item } \\
\text { response data } \\
\text { to identify } \\
\text { PTSD }\end{array}$ & $\begin{array}{l}\text { Written } \\
\text { text, survey } \\
\text { responses }\end{array}$ & $\begin{array}{l}\text { Item response } \\
\text { theory and } \\
\text { text } \\
\text { classification }\end{array}$ & - & $\begin{array}{l}\text { Posttraumat } \\
\text { ic stress } \\
\text { disorder }\end{array}$ & $\begin{array}{l}\text { Combining text classification and } \\
\text { item response theory models } \\
\text { provides an efficient approach to } \\
\text { estimating the latent trait. }\end{array}$ \\
\hline $\begin{array}{l}\text { Shin et al. } \\
\text { (2019) }\end{array}$ & $\begin{array}{l}\text { Topic } \\
\text { modeling for } \\
\text { item distractor } \\
\text { generation }\end{array}$ & Written text & $\begin{array}{l}\text { Machine } \\
\text { learning }\end{array}$ & $\mathrm{OF}$ & $\begin{array}{l}\text { Knowledge } \\
\text { and skills in } \\
\text { biology }\end{array}$ & $\begin{array}{l}\text { Latent topic modeling supports } \\
\text { the identification of students' } \\
\text { misconceptions in biology and } \\
\text { aids the development of } \\
\text { distractors. }\end{array}$ \\
\hline
\end{tabular}


